\title{
Numerical Investigation on MHD Flow and Heat Transfer over an Exponentially Stretching Sheet with Viscous Dissipation and Radiation Effects
}

\author{
Inci Çilingir Süngü ${ }^{1}$ \\ ${ }^{1}$ Department of Mathematics, University of Ondokuz Mayis, Samsun, Turkiye
}

\begin{abstract}
This study is to examine the steady two dimensional laminar flow of a viscous incompressible electrically conducting fluid over a continuous surface. In this study DTM-Padé method is used to solve which is a combination of differential transform method (DTM) and Padé approximant. Comparisons between the solutions obtained by DTM and DTM-Padé and are shown that DTM-Padé is the completely powerful method then DTM for solving the problems in which boundary conditions at infinity. Also in this study the effect of Magnetic and Radiation parameters, Prandtl number and Eckert number for velocity and temperature distributions are investigated.
\end{abstract}

\section{Introduction}

The nonlinear equations have found numerous applications of Applied Mathematics, Physics and technological processes. Nonlinear equations cover the cases of the following types: surface waves incompressible fluids, hydro magnetic waves in cold plasma, acoustic waves in inharmonic crystal, plastic sheets, paper production and many others. However, these equations are difficult to be solved analytically and sometimes it is impossible then application must be made with relevant numerical methods such as shooting method, finite difference etc. The flow of a viscous incompressible fluid over a stretching sheet has many interests over 10 years and was firstly considered by Sakiadis [1]. After many authors were focused this problem with extended conditions/situations like: two dimensional flow over a stretching sheet problem [2], heat transfer in the flow over stretching surface [3], boundary layer problem over an exponentially stretching sheet with thermal radiation [4], magneto hydrodynamic (MHD) boundary layer flow of a viscous fluid over an exponentially stretching sheet with radiation effect [5], MHD flow and heat transfer over an exponentially stretching sheet with viscous dissipation and radiation effects[6],etc.

The differential transform method was used first by Zhou [7] who solved linear and nonlinear initial value problems in electric circuit analysis. This method constructs an analytical solution in the form of a polynomial. The differential transform is well suited to Corresponding Author : incicilingir@gmail.com 
combine with other numerical techniques. The main advantages of these hybrid methods are that they can be used to solve both linear and nonlinear equations without linearization. But DTM solutions are not enough to wide region problem. In these domains one should use multi-step DTM or another combine. DTM and Padé approximant was used to solve MHD flow problems in [8-11]. In this study the same problem with [6] which was solved numerically with Runge-Kutta method is considered and solved analytically with Differential Transform (DTM)-Padé method. The validity of the proposed approach has been confirmed by comparing the results [4- 6].

\section{Mathematical Formulation}

\subsection{Governing Equations and Boundary Conditions}

Consider the two-dimensional steady flow of viscous incompressible electrically conducting fluid over a continuous stretching sheet at $y=0$. A uniform magnetic field of strength $B_{0}$ has been applied to perpendicular to the stretching sheet. The induced magnetic field is neglected. We have the momentum equation and energy equation as follows,

$$
\begin{gathered}
u \frac{\partial u}{\partial x}+v \frac{\partial u}{\partial y}=v \frac{\partial^{2} u}{\partial y^{2}}-\frac{\sigma B_{0}}{\rho} u \\
\rho c_{p}\left(u \frac{\partial T}{\partial x}+v \frac{\partial T}{\partial y}\right)=\kappa \frac{\partial^{2} T}{\partial y^{2}}-\frac{\partial q_{r}}{\partial y}+\mu\left(\frac{\partial u}{\partial y}\right)^{2}+\sigma B_{0}{ }^{2} u^{2}
\end{gathered}
$$

along with the incompressibility condition

$$
\frac{\partial u}{\partial x}+\frac{\partial v}{\partial y}=0
$$

Here $u$ and $v$ are the velocity components in $x$ and $y$ directions, respectively, $\rho$ is the density, $\mu$ and $v\left(=\frac{\mu}{\rho}\right)$ are dynamic and kinematic viscosity, respectively, $c_{p}$ is the specific heat, $q_{r}$ is the radiative heat flux, $\kappa$ is the thermal conductivity and $T$ is temperature.

The boundary conditions are 


$$
\begin{aligned}
& u(0)=U_{0} e^{x / L} \quad v(0)=0 \quad T(0)=T_{\infty}+T_{0} e^{x / 2 L} \\
& \text { for } y \rightarrow \infty \quad u \rightarrow 0 \quad T \rightarrow 0
\end{aligned}
$$

where $U_{0}$ is the reference velocity, $T_{0}$ is the temperature at the plate and $T_{\infty}$ is the temperature far away from the plate while $L$ is the reference length.

The radiative heat flux $q_{r}$ is

$$
q_{r}=-\frac{4 \sigma^{*}}{3 k^{*}} \frac{\partial T^{4}}{\partial y}
$$

[12]. Here $k^{*}$ is the mean absorption coefficient and $\sigma^{*}$ is the Stefan_Boltzman constant. $T^{4}$ is expressed as a linear function of temperature, hence

$$
T^{4} \cong 3 T_{\infty}^{3} T-3 T_{\infty}^{4}
$$

is obtained by Taylor series at $T_{\infty}$ neglecting higher order terms.

Using equations (5) and (6) in equation (2) heat transport equation is given as

$$
\rho c_{p}\left(u \frac{\partial T}{\partial x}+v \frac{\partial T}{\partial y}\right)=\left(\kappa+\frac{16 \sigma^{*} T_{\infty}^{3}}{3 k^{*}}\right) \frac{\partial^{2} T}{\partial y^{2}}+\mu \frac{\partial^{2} T}{\partial y^{2}}+\sigma B_{0}{ }^{2} u^{2}
$$

Using the similarity variables

$$
\begin{gathered}
u=U_{0} e^{x / L} f^{\prime}(\eta) \quad, v=-\sqrt{\frac{v U_{0}}{2 L}} e^{x / 2 L}\left\{f(\eta)+\eta f^{\prime}(\eta)\right\} \\
T=T_{0} e^{x / 2 L} \theta(\eta)+T_{\infty}
\end{gathered},
$$

equations (1), (2) and (7) are reduced to

$$
f^{\prime \prime \prime}-2\left(f^{\prime}\right)^{2}+f f^{\prime \prime}-M f^{\prime}=0
$$




$$
\left(1+\frac{4}{3} R\right) \theta^{\prime \prime}+\operatorname{Pr}\left(f \theta^{\prime}-f^{\prime} \theta+E c\left(f^{\prime \prime}\right)^{2}+M \cdot E c\left(f^{\prime}\right)^{2}\right)=0
$$

with the boundary conditions

$$
\begin{array}{lll}
f(0)=0 \quad f^{\prime}(0)=1 & \theta(0)=1 \\
\text { for } \eta \rightarrow \infty & f^{\prime} \rightarrow 0 & \theta \rightarrow 0
\end{array}
$$

Here the primes denote differentiation with respect to $\eta$ and dimensionless parameters are

$$
\begin{array}{lll}
M=\frac{2 \sigma B_{0}{ }^{2} L}{\rho U_{0} e^{x / L}} & : & \text { Magnetic Parameter } \\
E c=\frac{U_{0}{ }^{2}}{T_{0} c_{p}} & : & \text { Eckert Number } \\
\operatorname{Pr}=\frac{\mu c_{p}}{\kappa} & : & \text { Prandtl Number } \\
R=\frac{4 \sigma^{*} T_{\infty}^{3}}{k^{*} \kappa} & : & \text { Radiation Parameter. }
\end{array}
$$

\subsection{DTM with the Padé Approximant}

In the following, we introduce the main features of the differential transformation method according to the differential transform of the $n$th derivative of a function $y(x)$ in one variable is defined as follows:

$$
Y(n)=\frac{1}{n !}\left[\frac{d^{n} y(x)}{d x^{n}}\right]_{x=x_{0}} .
$$

In Equation (12), $y(x)$ is the original function and $Y(n)$ is the transformed function and the differential inverse transform of $Y(n)$ is defined as follows:

$$
y(x)=\sum_{n=0}^{\infty} Y(n)\left(x-x_{0}\right)^{n} .
$$


In real applications, function $y(x)$ is expressed by a finite series and Equation (13) can be written as

$$
y(x)=\sum_{n=0}^{i} Y(n)\left(x-x_{0}\right)^{n}
$$

From Equation (14), we obtain

$$
y(x)=\sum_{n=0}^{\infty} \frac{\left(x-x_{0}\right)^{n}}{n !}\left[\frac{d^{n} y(x)}{d x^{n}}\right]_{x=x_{0}} .
$$

Actually Equations (15) implies that the concept of differential transform is derived from Taylor series expansion. Although DTM is not able to evaluate the derivatives symbolically, relative derivatives can be calculated by an iterative way which is described by the transformed equations of the original function. DTM is a semi-analytic method due to equation (15) implies $y(x)=\sum_{n=i+1}^{\infty} Y(n)\left(x-x_{0}\right)^{n}$ is negligibly small. In fact $i$ is decided by the convergence of natural frequency. DTM construct a series solution which is actually a truncated series solution. This series solution gives a good approximation to the true solution in a very small region. But it has slow convergent rate or completely divergent in the wider region.

Padé approximant is the approximation of a function by a rational function of given order. In this technique, we find a polynomial of degree $p+q+1$ as a rational function which is a polynomial of degree $p$ divided by a polynomial of degree $q$. This gives often better approximation of the function than truncating its Taylor expansion, especially for a function has conditions at infinity.

Taking the differential transform of equations (9) and (10), we have

$$
\begin{aligned}
& (n+3)(n+2)(n+1) F(n+3)-2 \sum_{n_{1}=0}^{n}\left(n_{1}+1\right)\left(n-n_{1}+1\right) F\left(n_{1}+1\right) F\left(n-n_{1}+1\right) \\
& +\sum_{n_{1}=0}^{n}\left(n-n_{1}+1\right)\left(n-n_{1}+2\right) F\left(n_{1}\right) F\left(n-n_{1}+2\right)-M(n+1) F(n+1)=0
\end{aligned}
$$




$$
\begin{aligned}
& \left(1+\frac{4}{3} R\right)(n+2)(n+1) T(n+2)+\operatorname{Pr} \sum_{n_{1}=0}^{n}\left(n-n_{1}+1\right) F\left(n_{1}\right) T\left(n-n_{1}+1\right) \\
& -\operatorname{Pr} \sum_{n_{1}=0}^{n}\left(n_{1}+1\right) F\left(n_{1}+1\right) T\left(n-n_{1}\right) \\
& +\operatorname{Pr} . \text { Ec. } \sum_{n_{1}=0}^{n}\left(n_{1}+2\right)\left(n_{1}+1\right) F\left(n_{1}+2\right)\left(n-n_{1}+2\right)\left(n-n_{1}+1\right) F\left(n-n_{1}+2\right) \\
& +\operatorname{Pr} \text {.Ec.M } \sum_{n_{1}=0}^{n}\left(n_{1}+1\right)\left(n-n_{1}+1\right) F\left(n_{1}+1\right) F\left(n-n_{1}+1\right)=0
\end{aligned}
$$

Where $F(n)$ and $T(n)$ are the differential transform of $f(\eta)$ and $\theta(\eta)$, respectively.

The boundary conditions are rewritten as initial conditions:

$$
f(0)=0 \quad f^{\prime}(0)=1 \quad \theta(0)=1 \quad f^{\prime \prime}(0)=\alpha \quad \theta^{\prime}(0)=\beta
$$

The differential transformations of conditions in (18) are given as

$$
F(0)=0 \quad F(1)=1 \quad T(0)=1 \quad F(2)=\frac{\alpha}{2} \quad T(1)=\beta
$$

where $\alpha$ and $\beta$ are to be determined.

Using the conditions (19) in the recurrence relations (16) and (17) leads to the solution of a system of algebraic equations. After finding the DTM solutions for velocity and heat transfer, the Padé approximant must be applied to obtain $\alpha$ and $\beta$. To find the values of $\alpha$ and $\beta$ it is taken limit tends to infinity of the Padé approximant of DTM solution.

\section{RESULTS AND DISCUSSION}

In this study all computations are done with Maple. In the DTM solution 20 term are taken so that the order of Padé approximant is [10,10]. Skin friction coefficient and local Nusselt number are determined as $f^{\prime \prime}(0)=\alpha$ and $-\theta^{\prime}(0)=-\beta$, respectively. The shear stress which is proportional to $\alpha$ and the values of heat transfer coefficient $\beta$ are given in Table 1 and Table 2 respectively for various values of parameters. 
Table 1, $\alpha$ values for different values of Magnetic parameter

\begin{tabular}{|c|c|c|c|c|}
\hline & $\mathrm{M}=0$ & $\mathrm{M}=1 / 2$ & $\mathrm{M}=1$ & $\mathrm{M}=2$ \\
\hline$\alpha=f^{\prime \prime}(0)$ & -1.284989678 & -1.466585678 & -1.628654619 & -1.912448326 \\
\hline
\end{tabular}

Table 2, $\beta$ values for different values of Magnetic parameter, Radiation Parameter and Eckert number

\begin{tabular}{|c|c|c|c|c|c|c|c|}
\hline$\beta=\theta^{\prime}(0)$ & Ec & $\operatorname{Pr}$ & $\mathrm{R}$ & $\mathrm{M}=0$ & $\mathrm{M}=1 / 2$ & $\mathrm{M}=1$ & $\mathrm{M}=2$ \\
\hline & \multirow{3}{*}{0} & \multirow{3}{*}{1} & 0 & -.9652361600 & -.9120918338 & -.8577114599 & -.8073383447 \\
\hline & & & 0.5 & -.6765622412 & -.6286223821 & -.5881375790 & -.5539571142 \\
\hline & & & 1 & -.5389970000 & -.5149450441 & -.4620199464 & -.4068818952 \\
\hline & \multirow{3}{*}{0.2} & \multirow{3}{*}{1} & 0 & -.8712600289 & -.7573788555 & -.6713115526 & -.5445636315 \\
\hline & & & 0.5 & -.6223039456 & -.5471632796 & -.4871645113 & -.3895030707 \\
\hline & & & 1 & -.4937037509 & -.4444687547 & -.3832084466 & -.3265448806 \\
\hline
\end{tabular}

From the equation (16), it is seen that variations in $\mathrm{Pr}, \mathrm{R}$ and $\mathrm{Ec}$ do not affect the value of $\alpha$. It is observed from the Table 1 that surface shear stress $(\alpha)$ decreases as Magnetic Parameter increases. Table 2 indicates that an increase of parameters R, Ec and M increases the value of heat transfer coefficient $\beta$. To describe the flow behavior we consider the profiles of $f^{\prime}(\eta)$ and $\theta(\eta)$. For the values of $\operatorname{Pr}=1, \mathrm{M}=1, \mathrm{Ec}=0.2$ and $\mathrm{R}=0$ , the velocity profiles $f^{\prime}(\eta), f(\eta)$ and the temperature profile $\theta(\eta)$ are shown in Figure 1. As seen that $f^{\prime}(\eta)$ and $f(\eta)$ are inversely proportional to each other. 
$M=1 \quad P_{x}=1 \quad E_{c}=0.2 \quad R=0$

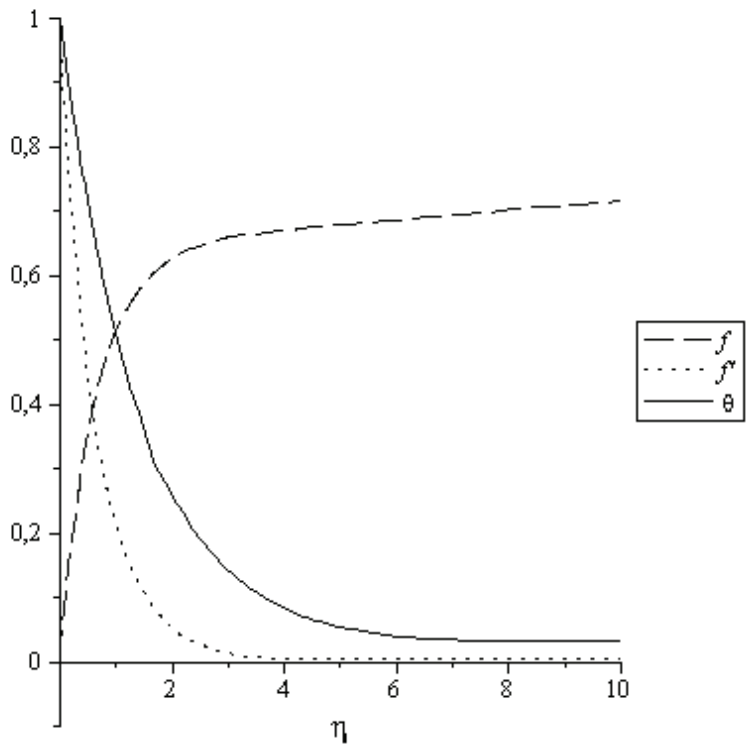

Figure 1. The velocity profiles $f^{\prime}(\eta), f(\eta)$ and the temperature profile $\theta(\eta)$ at $\operatorname{Pr}=1$, $\mathrm{Ec}=0.2, \mathrm{R}=0, \mathrm{M}=1$.

The comparisons of the solutions of DTM with the DTM-Padé are shown in Figure

2. DTM is an analytical method to solve initial value problem in a small region. Figure 2 shows that the solution obtained from DTM is only valid for small values of $\eta$ and does not satisfy the boundary condition at infinity. Further, DTM-Padé approximation is shown to yield a convergent solution at infinity and agree very well with literature [4-6]. 


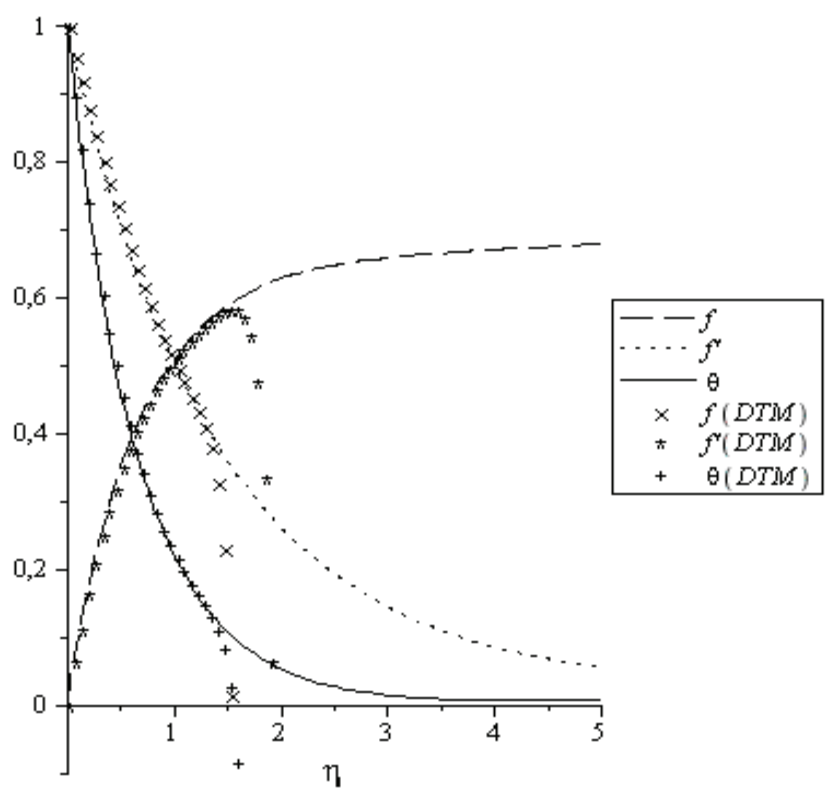

Figure 2. DTM and DTM-Padé solutions of velocity profile $f^{\prime}(\eta)$ and $\theta(\eta)$ for $\operatorname{Pr}=1$, $\mathrm{Ec}=0.2, \mathrm{R}=0, \mathrm{M}=1$.

The influence of the magnetic parameter $\mathrm{M}$ on the velocity profile $f^{\prime}(\eta)$ is shown in Figure 3. This figure indicates a decrease in the magnetic parameter $\mathrm{M}$ by choosing the fixed $\operatorname{Pr}=1, \mathrm{Ec}=0$ and $\mathrm{R}=0$ that corresponds to an increase in the velocity boundary layer thickness. In figure 4 , it is shown that the temperature profile $\theta(\eta)$ for various values of magnetic parameter $M$. The increase in magnetic Parameter $M$ causes the increase in temperature profile. 


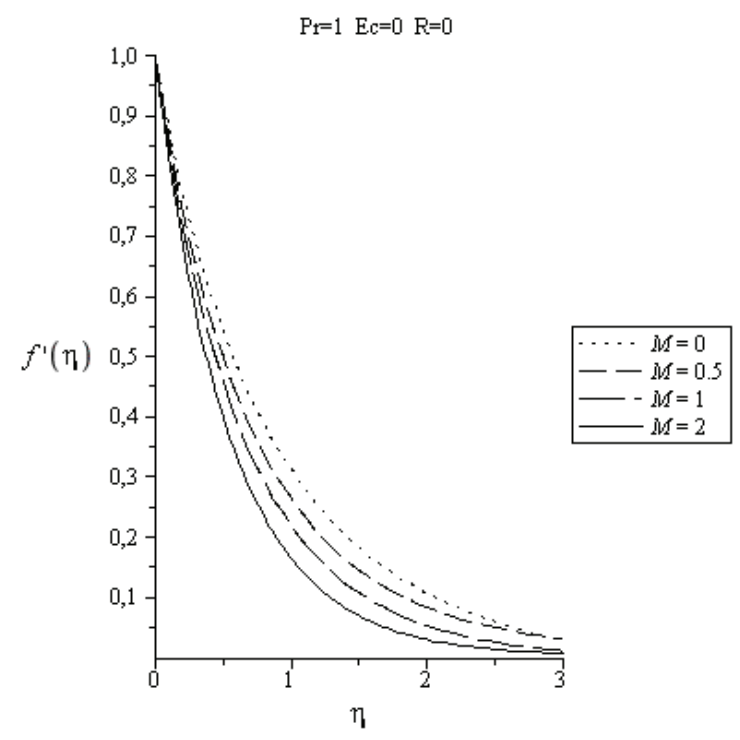

Figure 3. Velocity profile $f^{\prime}(\eta)$ for different $M$ values at $\mathrm{R}=0$.

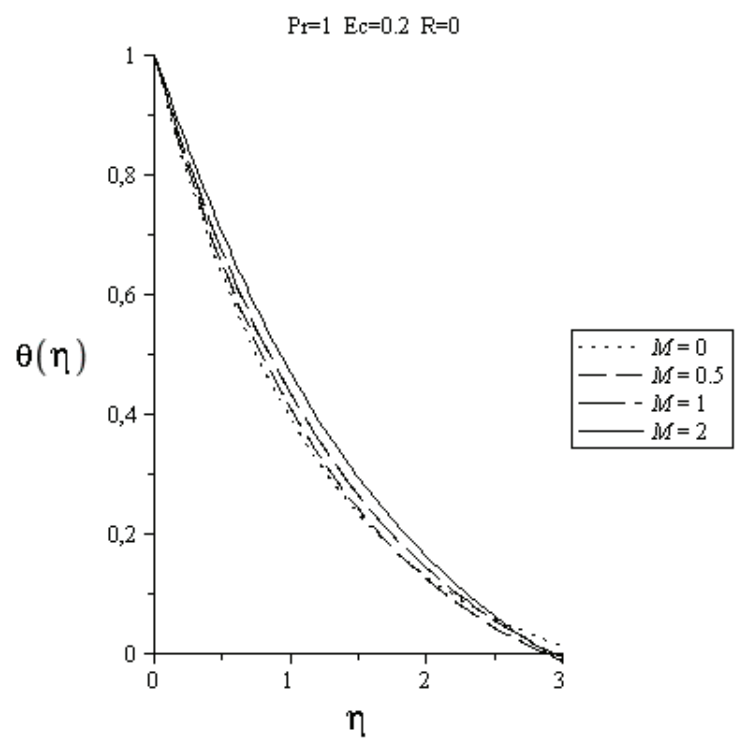

Figure 4. Temperature profile $\theta(\eta)$ for different $\mathrm{M}$ values at $\mathrm{Pr}=1, \mathrm{Ec}=0.2, \mathrm{R}=0$.

From Figures 5and 6, it is seen that the effects of Radiation Parameter and Eckert Number on the temperature profile $\theta(\eta)$. The temperature profiles and the thermal boundary layer thickness increase with the increase of Ec and R. 


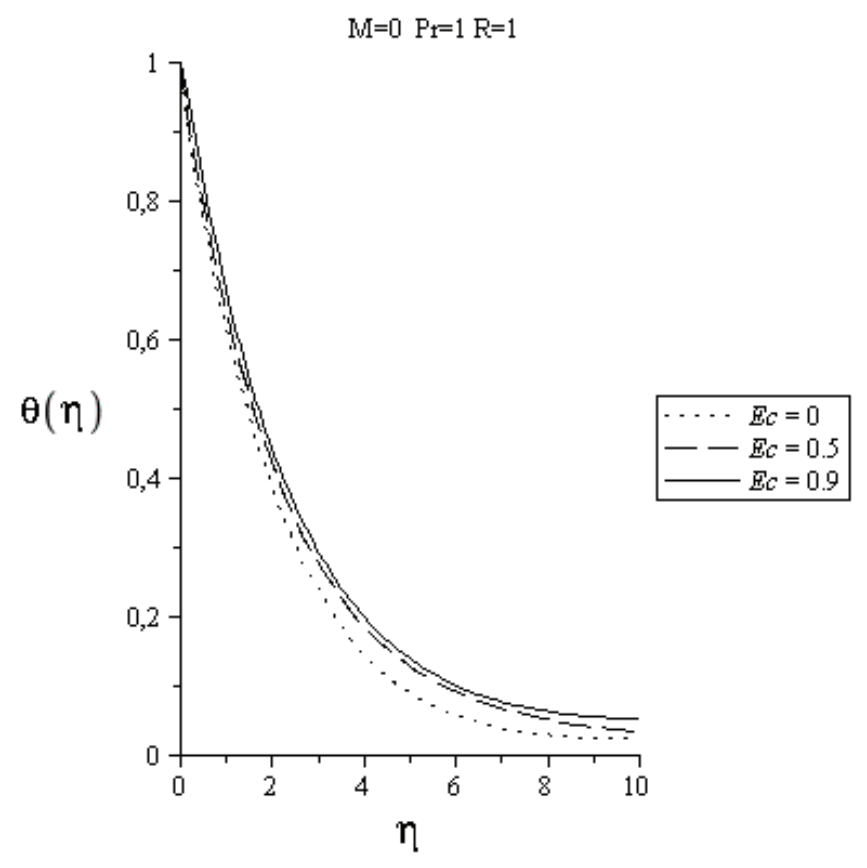

Figure 5. Temperature Profile $\theta(\eta)$ for different Ec values.

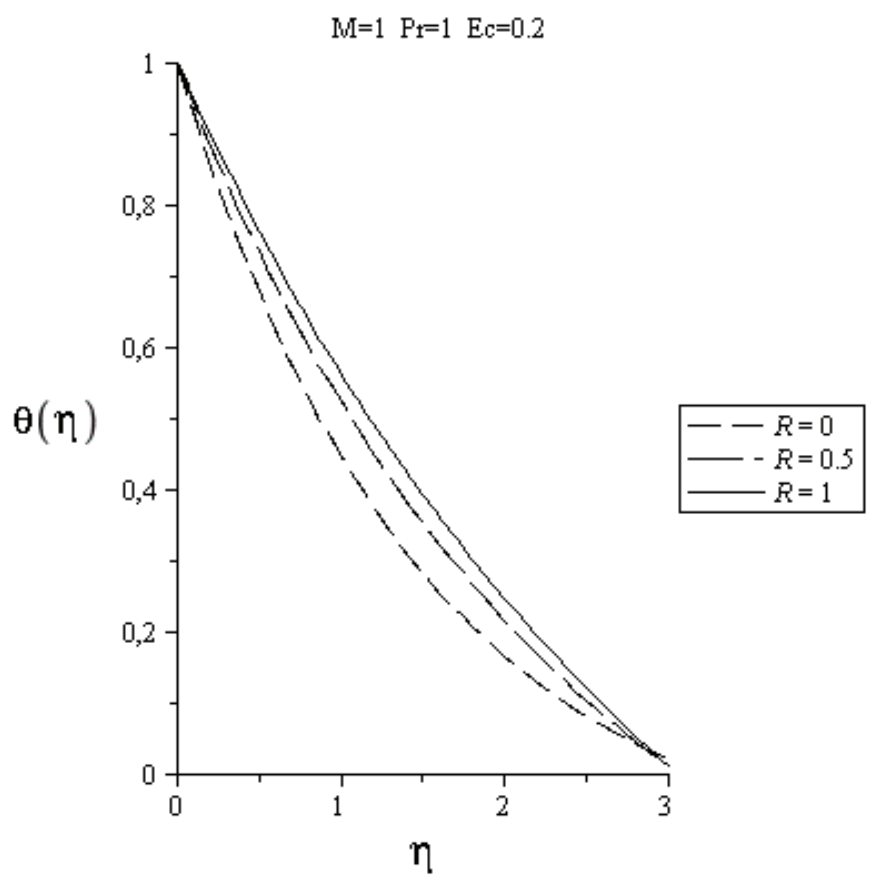

Figure 6. Temperature Profile $\theta(\eta)$ for different $\mathrm{R}$ values. 
On the other hand, it has been observed in Figure 7 that the temperature profile decreases with an increase of the Pr. All these results are coincide with the literature.

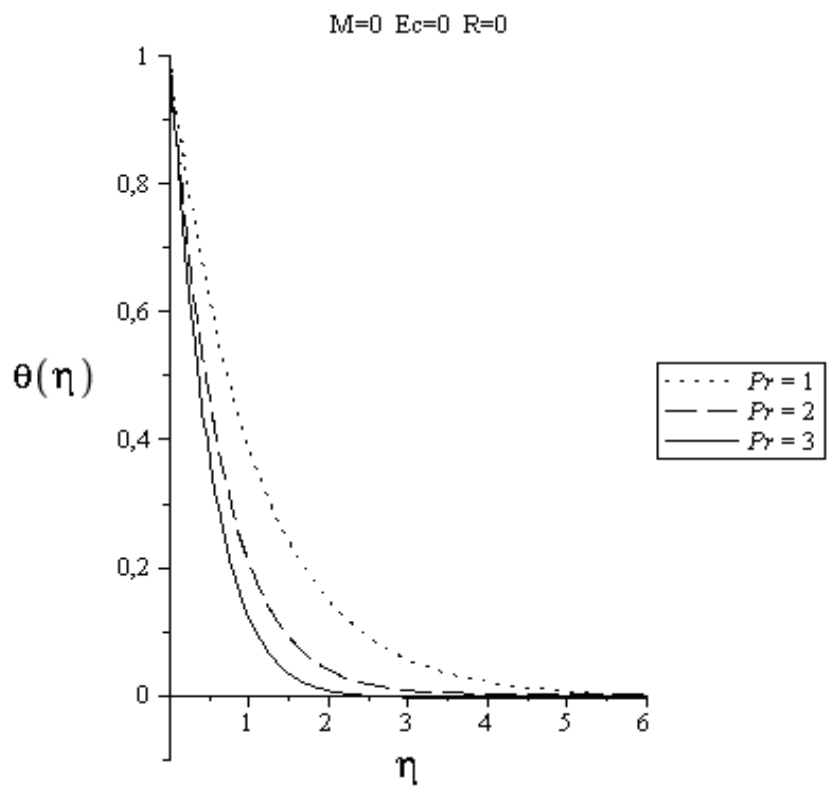

Figure 7. Temperature profile $\theta(\eta)$ for different $\operatorname{Pr}$ values.

\section{CONCLUSION}

In this work DTM-Padé is used to find analytical solution of boundary layer flow over an exponentially stretching sheet with thermal radiation. The comparison of the solution obtained from DTM and DTM-Padé are given by graphically. DTM is a convenient way to solve fluid equations because of the symbolic computation. But, for a wide region it is not enough to get a convergent solution. With using symbolic computation one can have a valid solution with DTM-Padé method. DTM-Padé is easy to implement and effective method for the problems on an unbound domain, when compared DTM.

\section{REFERENCES}

[1] B.C. Sakiadis, Boundary-layer behavior on continuous solid surfaces: I. Boundarylayer equations for two-dimensional and axi-symmetric flow, AIChE Journal, 7, 2628,(1961).

[2] L.J. Crane, Flow past a stretching sheet, JAMP, 21, 645-647, (1970).

[3] P.S. Gupta, A.S. Gupta, Heat and mass transfer on a stretching sheet with suction or blowing, Can. J. Chem. Eng, 55, 744-746, (1967). 
[4] B. Bidin, R. Nazar, Numerical solution of the boundary layer flow over an exponentially stretching sheet with thermal radiation. Eur. J. Appl. Eng. Sci. Res., 33(4), 710-717, (2009).

[5] A. Ishak, MHD boundary layer flow due to an exponentially stretching sheet with radiation effect, Sains Malays., 40, 391-395, (2011).

[6] R. N. Jat, G. Chand, MHD Flow and Heat Transfer over an Exponentially Stretching Sheet with Viscous Dissipation and Radiation Effects, Applied Mathematical Sciences, 7, 4, $167-180,(2013)$.

[7] J.K. Zhou, Differential Transformation and its Applications for Electrical Circuits, Huazhong University Press, Wuhan, P. R. China, In Chinese.

[8] M.M. Rashidi, T. Hayat, M. Keimanesh, H. Yousefian, A Study on Heat Transfer in a Second-Grade Fluid Through a Porous Medium with the Modified Differential Transform Method, Heat Transfer-Asian Research, 42, 31-45,(2013).

[9] M. Azimi, D. D. Ganji, F. Abbassi, Study on MHD Viscous Flow over a Stretching Sheet Using DTM-Pade’ Technique, Mod. Mech. Eng., 2 ,126-129, (2012).

[10] M. Thiagarajan, K. Senthilkumar, DTM-Padé Approximants for MHD Flow with Suction/Blowing JAFM, 6, 4, 537-543,(2013).

[11] O. Anwar Bég, M.M. Rashidi, M.T. Rastegari, T. A. Bég, S.S. Motsa and A. Halim, DTM-Padé Numerical Simulation of Electro hydrodynamic Ion Drag Medical Pumps with Electrical Hartmann and Electrical Reynolds Number Effects, J. Adv. Biotechnol. Bioeng., 1, 62-79, (2013).

[12] M. Sajid, T. Hayat, Influence of thermal radiation on the boundary layer flow due to an exponentially stretching sheet, Int. Commun. Heat Mass Transfer, 3,5,347-356, (2008). 